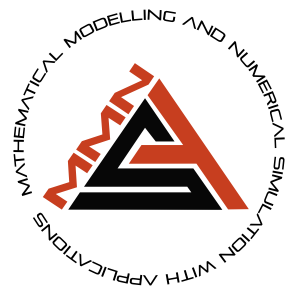

\title{
Bi-dimensional crime model based on anomalous diffusion with law enforcement effect
}

\author{
Francisco Javier Martínez-Farías 1,*, ${ }^{\ddagger}$, Anahí Alvarado-Sánchez ${ }^{2,{ }^{\ddagger}}$, Eduardo \\ Rangel-Cortes @1, ${ }^{\ddagger}$ and Arturo Hernández-Hernández $\odot 1,{ }^{\ddagger}$
}

\author{
${ }^{1}$ Escuela Superior de Apan, Universidad Autónoma del Estado de Hidalgo, Carretera Apan-Calpulalpan Km 8, Col. \\ Chimalpa, C.P 43920, Apan, Hidalgo, México, ${ }^{2}$ Instituto de Ciencias Nucleares, Universidad Nacional Autónoma de \\ México, Apdo. Postal 70-543, 04510, Cd.Mx., México \\ *Corresponding Author \\ †francisco_martinez@uaeh.edu.mx (Francisco Javier Martínez-Farías); anahi_a.s@ciencias.unam.mx (Anahí Alvarado-Sánchez); \\ eduardo_rangel@uaeh.edu.mx (Eduardo Rangel-Cortes); arturo_hernandez@uaeh.edu.mx (Arturo Hernández-Hernández)
}

\begin{abstract}
Several models based on discrete and continuous fields have been proposed to comprehend residential criminal dynamics. This study introduces a two-dimensional model to describe residential burglaries diffusion, employing Lévy flights dynamics. A continuous model is presented, introducing bidimensional fractional operator diffusion and its differences with the 1-dimensional case. Our results show, graphically, the hotspot's existence solution in a 2-dimensional attractiveness field, even fractional derivative order is modified. We also provide qualitative evidence that steady-state approximation in one dimension by series expansion is insufficient to capture similar original system behavior. At least for the case where series coefficients have a linear relationship with derivative order. Our results show, graphically, the hotspot's existence solution in a 2-dimensional attractiveness field, even if fractional derivative order is modified. Two dynamic regimes emerge in maximum and total attractiveness magnitude as a result of fractional derivative changes, these regimes can be understood as considerations about different urban environments. Finally, we add a Law enforcement component, embodying the "Cops on dots" strategy; in the Laplacian diffusion dynamic, global attractiveness levels are significantly reduced by Cops on dots policy but lose efficacy in Lévy flight-based diffusion regimen. The four-step Preditor-Corrector method is used for numerical integration, and the fractional operator is approximated, getting the advantage of the spectral methods to approximate spatial derivatives in two dimensions.
\end{abstract}

Key words: Residential burglary; Lévy flights; fractional operator; anomalous diffusion; hotspots; law enforcement AMS 2020 Classification: 60K50;26A33;34D20

\section{Introduction}

The present work is motivated by the impact that insecurity produces for an urban area; evidently, there are different types of crimes, and each one must be studied to later design prevention policies. This work studies criminal agents' displacement effect, specialized in house robbery, with the possibility of making long journeys in a short time, described by Lévy Flights in a two-dimensional environment. We consider that this way of describing criminal diffusion is more realistic than models based on conventional diffusion. Understanding the mobility of certain social groups within an urban area is of great relevance for policymakers, especially displacements at specific geographical locations detrimental to security and forming specific patterns [1]. Several of today's models focus on the displacement of residential burglaries, this from the pioneering work by Short et al. [2, 3]. Both approximations show more significant criminal activity areas, known as hotspots. This work inspired numerous modifications, generalizations, and theoretical studies that described dynamical

> Received: 15.12.2021 > Revised: 21.01.2022 > Accepted: 22.01.2022 > Published: 27.01.2022 
properties, as in references $[4,5,6,7,8,9,10,11,12,13]$. In these models, a local random walk with a certain degree of statistical bias defines diffusion dynamics among the criminal population. The core of model dynamics is that home thief's agents have a higher stochastical preference for specific targets known as the highest attractive zones. Several studies have been used to assess the stability of certain kinds of solutions to crime phenomena, as presented in references [12,13,14, 15]. This dynamic belongs to the so-called reaction-diffusion models, which are known to comprise a broad set of spatial distribution patterns [16]. Biased Brownian motion displacement is the kernel of the agent version when the agents are sensitive to environmental gradients. In the continuum limit, this phenomenon is modeled by of cross-diffusion equation. It is represented by Keller-Segel operator [17, 18]. One of the cross-diffusion attributes is that the increments represent a local displacement, where the criminal agent moves from site $i$ to site $j$, and $j$ is in the neighborhood of $i$. A more general model allows agents to travel to sites outside the neighborhood. These models are known as non-local diffusion and belong to the so-called anomalous diffusion models.

A non-local diffusion model would assume that criminals can make long jumps in a short time, thus moving towards more attractive areas. For example, motorized mobility is an influencing factor. The cause that house burglars would incur greater risk when leaving a familiar area may be due to real-time information received by other thieves. Sharing/receiving this information dynamically with other criminals they compete with may seem unlikely. However, Calvo et al. [19] provide an analysis of conditions under which different criminal agents are likely to collaborate. According to this, it is established that, once a home robbery agent has moved away to a specific area, he again uses random walking as a strategy to locate a target. Chaturapruek et al. [6] propose that so-called Lévy flights can describe that above dynamic, where the probability distribution of jumps length of robbery agents follows an inverse power law distribution. Thus, criminal agents can move from site $i$ to $j$, where $j$ is no longer part of the $i$ neighborhood. Considering continuous limit, the fractional Laplacian operator appears analogously to the fractional Gierer Meinhardt model [20]. A particular property of fractional operator hotspots solutions is that they decay algebraically. Chaohao et al. [11] show that if the jump length is truncated, then a version of the conventional Laplacian diffusion model is obtained. It is considered that criminal agents do not leave a specific area, and the unique modification occurs in the diffusion coefficient. That study is carried out in one dimension for discrete and continuous cases; it also incorporates police effects on the criminal's attractiveness perception. S. Crúz-García et al. [21] propose an alternative method to Lévy flights, applying stochastic interference to the Jones et al. model [4], which contemplates large jumps from a small set of criminal agents at each time step. S. Crúz also found that if police presence is increased numerically in central hotspots, they will fragment into smaller areas. Other studies are based on discrete agent algorithms that incorporate Lévy flights in two dimensions and analyze patterns formation, depending on the model's parameters [22].

The police dissuasive influence is a multifaceted problem, and there are various proposals to be addressed, depending on the environmental conditions and the police agency's resources [23]. To illustrate this, the work of Jones [4] shows how police presence affects attractiveness when this is incorporated into the law enforcement scheme. Jones analyzes different strategies, among which Cops on the dots and Peripheral interdiction stand out; results from these studies depend on the urban environment's characteristics. Camacho [9] also compares these two strategies with one based on region partitioning into smaller areas (beats), within which cops can move, although they are not able to cross borders. Law enforcement has also been incorporated into one-dimensional fractional diffusion [6], based on the cops on the dots strategy; however, the parameter of criminal density diffusion are modified. In his work, Chaohao [11] also incorporates two forms of police agent's motion, one governed by biased Brownian diffusion and the second by Lévy flights. The main difference is that police shifts based on Lévy flights reach the steady-state solution in a shorter time.

The present work shows a two-dimensional extension of the Chaturapruek continuous model, incorporating law enforcement with Cops on dots strategy. These models have been published at the agent level (discrete) for the two-dimensional case, using Lévy flights, for example Brantingham et. al. [1], but this work proposes a deduction for the continuous two-dimensional case based on the one already made by Chaturapruek for one dimension. An interesting aspect is that it appears in our deduction, it is a new function that is the equivalent of Riemman's $\zeta$ for two dimensions, and that modifies the criminal diffusion coefficient. The main aim is to show numerically that hotspot solutions in attractiveness bi-dimensional fields are preserved by varying derivative order (in not truncated Lévy flights) using a spectral approximation to bi-dimensional fractional derivative operator. However, they may change shape or intensity. We also present criminal population spatial distribution patterns that reveal a more complex dynamic than the attractiveness field and exhibit strong dependence on the fractional order. The Cops on the dots strategy assumes that police officers have restricted movements, this differentiates the modeling behavior between house burglars with law enforcement. The investigatory police, who work within broader limits when applying their authority, represent a group that Lévy's flights might better model. However, in the present work, we do not consider this approach.

This paper is divided as follows; in Section 2, we introduce a model deduction for a two-dimensional problem. Subsection 2 presents the numerical method for model integration. Section 3 presents a qualitative analysis for stationary solutions, using a simplified attractiveness version in one dimension. A numerical sensitivity analysis of the model concerning the derivative order is performed in Section 4 . In Section 5 , we deduce the component that models law enforcement and present numerical results by changing fractional order, holding the Cops on dots strategy. In the final of this manuscript, a nomenclature section is presented.

\section{Continuum fractional model formulation}

The two-dimensional model is deduced regarding an analogous formulation of a one-dimension problem by Chaturapruek [6]. The scenario occurs in a lattice $\Omega$ with size $N \times N$, and the lattice spacing is $l=1 / N$. To simplify, we initiate the model derivation without including law enforcement. The position of each site $d$ in $\Omega$ is represented by $d=\left(d_{1}, d_{2}\right) \in \mathbb{R}^{2}$. At site $d$ and time $t$, there is an attractiveness $A_{d}(t)$, which is made of two components, $A_{d}(t)=A_{d}^{0}+B_{d}(t)$, where $A_{d}^{0}$ is the intrinsic attractiveness and $B_{d}(t)$ is time-dependent attractiveness. The evolution of $A_{d}(t)$ depends on whatever occurs around it, i.e., a criminal agent can change attractiveness in a time interval $\delta t$ by deciding whether or not to attack a $d$ site, and he does so with the probability

$$
p_{d}(t)=\frac{\epsilon A_{d}(t)}{1+\epsilon A_{d}(t)}
$$


where $\epsilon>0$ represents the effectiveness of the attractiveness at site $d$. Therefore, an increase in $B_{d}$ in the time interval $\delta t$ depends on $E_{d}(t)$, which indicates the number of criminal attacks on the same site during the same time interval

$$
B_{d}(t+\delta t)=B_{d}(t)+\theta E_{d}(t)
$$

here $\theta$ is the enhancement in attractiveness for a single criminal agent. Without considering that a criminal attack can exert influence in neighborhood (broken window effect [24]), the expression for $B_{d}(t)$ is

$$
B_{d}(t+\delta t)=B_{d}(t)(1-\omega \delta t)+\theta E_{d}(t)
$$

where $\omega$ represents attractiveness decay rate. Permitting the inclusion of the diffusive term for attractiveness, we have

$$
B_{d}(t+\delta t)=\left[\left(1-\eta^{*}\right) B_{d}(t)+\frac{\eta^{*}}{d^{\prime}} \sum_{d^{\prime}} B_{d^{\prime}}(t)\right](1-\omega \delta t)+\theta n_{d}(t) p_{d}(t)
$$

here $\eta^{*}>0$ represents of attractiveness influence in position $d$ to its immediate neighbors $d^{\prime}$, for this work, a regular Cartesian lattice is used, with $d^{\prime}=4$. Therefore, replacing in Eq. (2) the number of criminal attacks on site $d$ in the time interval $\delta t$ with the number of criminals $n_{d}(t)$, also replacing the probability that they will attack $p_{d}(t)$ and take the limit $l, \delta t \rightarrow 0$, keeping fixed the radius $l^{2} / \delta t$, and a new parameter $\epsilon^{*}=\theta \delta t$, which represents the influence of the criminal presence in each position $d$, in a time interval $(t, t+\delta t)($ the detailed derivation can be seen in the work of Short et al. [2]), we have

$$
\frac{\partial B(\mathbf{x}, t)}{\partial t}=\frac{\eta^{*} l^{2}}{4 \delta t} \Delta B(\mathbf{x}, t)-\omega B(\mathbf{x}, t)+\epsilon^{*} \frac{l^{2}}{\delta t} \rho(\mathbf{x}, t) A(\mathbf{x}, t),
$$

where $\rho(\mathbf{x}, t)=\lim _{l \rightarrow 0} n_{d}(t) / l^{2}$ is the criminal density, and the position $\mathbf{x} \in[0,1] \times[0,1]$ is defined as

$$
\mathbf{x}=\left(x_{1}, x_{2}\right)=\lim _{N \rightarrow \infty, l \rightarrow 0}\left(d_{1} l, d_{2} l\right), d_{1}, d_{2} \in[1, N]
$$

For modeling criminal displacement agents in 2-D, we have the probability that a criminal will arrive at site $d=\left(d_{1}, d_{2}\right)$ from $i=\left(i_{1}, i_{2}\right)$, analogously to that defined by Chaohao et al. [11] for 1-D

$$
q_{i \rightarrow d}(t)=\frac{w_{i \rightarrow d}}{\sum_{j \in \mathbb{Z}^{2}, j \neq i} w_{i \rightarrow j}},
$$

the relative weight $w_{i \rightarrow d}$ is defined as

$$
w_{i \rightarrow d}(t)= \begin{cases}\frac{A_{i}(t)}{l \mu \| i-d \mid \mu \mu}, & 1 \leq\|i-d\|<\infty \\ 0, & \text { other case }\end{cases}
$$

with || · || the Euclidean norm. Lévy flight is an anomalous diffusion, where the density function of jump length probability possesses an algebraic decay [20], so $\mu$ is the exponent of the underlying power law. Thus, can be expressed the following

$$
\sum_{j \in \mathbb{Z}^{2}, j \neq i} w_{i \rightarrow j}=\sum_{j \in \mathbb{Z}^{2}, j \neq i} \frac{A_{j}(t)}{|\mu||i-j| \mid \mu}=\sum_{j \in \mathbb{Z}^{2}, j \neq i} \frac{A_{j}(t)-A_{i}(t)}{|\mu||i-j| \mid \mu}+\sum_{j \in \mathbb{Z}^{2}, j \neq i} \frac{A_{i}(t)}{|\mu||i-j| \mid \mu} .
$$

On the other hand, bearing in mind the Riemann sum definition, on the continuum limit $l<<1$ for $D$ dimensions, the operator $\mathcal{L}$ can be expressed as

$$
\mathcal{L} f(\mathbf{x})=\frac{1}{l D} \int_{\mathbf{y} \in \mathbb{R}^{D}} \frac{f(\mathbf{y})-f(\mathbf{x})}{\|\mathbf{y}-\mathbf{x}\|^{\mu}} d \mathbf{y} \approx \sum_{d \in \mathbb{Z}^{D}, d \neq i} \frac{f(\mathbf{y})-f(\mathbf{x})}{|\mu| \mid \mathbf{y}-\mathbf{x} \|^{\mu}}
$$

Eq. (7) can be compared with respect to the fractional operator definition in $D$ dimensions [20]

$$
-(-\Delta)^{s} f(\mathbf{x})=C_{D, 2 s} \int_{\mathbb{R}^{D}} \frac{f(\mathbf{y})-f(\mathbf{x})}{\| \mathbf{y}-\left.\mathbf{x}||\right|^{D+2 s}} d \mathbf{y}, C_{D, 2 s}=2^{2 s} \frac{\Gamma((D+2 s) / 2)}{\pi^{D / 2}|\Gamma(-s)|}, 0<s,
$$

where $f(\mathbf{x}): \mathbb{R}^{D} \rightarrow \mathbb{R}$. To simplify notation, it is defined $-(-\Delta)^{s}=\Delta^{s}[6]$, therefore, is possible to relate $\mathcal{L}$ with $\Delta^{s}$ as follows

$$
\mathcal{L} f(\mathbf{x})=l^{-D} C_{D, 2 s} \Delta^{S} f(\mathbf{x}) \text {, and } \mu=D+2 s .
$$

For bi-dimensional case $(D=2)$ implies $\mu \in(2, \infty)$ ( 4 coincide with the usual Laplacian operator). For cases where the order of the derivative $s \in \mathcal{Z}$, the operator $\Delta^{s}$ is a local property (conventional differentiability) and loses this local property when $s$ is a non-integer [25]. A notable difference between cases 1 and 2-dimensions is explained in the following table:

Prefactors 2 and 4 in Table 1 are a consequence of the characteristic symmetries of the corresponding dimension. Non-integer values in the norm have their origin in diagonals that connect the point $i$ with $j$. 


\begin{tabular}{lll} 
Type of neighbors & $D=1$ & $D=2$ \\
$(i, j)$ & $|i-j|=$ & ||$i-j \|=$ \\
\hline first & 1 & $1, \sqrt{2}$ \\
second & 2 & $2, \sqrt{5}, 2 \sqrt{2}$ \\
third & 3 & $3, \sqrt{10}, \sqrt{13}, 3 \sqrt{2}$ \\
r-th & $r$ & $r, \sqrt{r^{2}+1^{2}}, \sqrt{r^{2}+2^{2}}, \ldots, r \sqrt{2}$ \\
\hline$\sum_{j \in \mathbb{Z}^{D}, j=i} \frac{1}{\|i-j\| \mu}=$ & $2 \sum_{r=1}^{\infty} \frac{1}{r^{\mu}}$ & $4 \sum_{r=1}^{\infty} \frac{1}{\left[\sum_{\alpha=0}^{r} \sqrt{\left(r^{2}+\alpha^{2}\right)}\right]^{\mu}}$
\end{tabular}

Table 1. The development in a series of accessible distances between the position $i$ with $j$ is shown.

Proposition 1 For $\mu \geq 2$ and $r \geq 1$ the following inequality is satisfied:

$$
\frac{1}{r^{\mu}} \geq \frac{1}{\left[\sum_{\alpha=0}^{r} \sqrt{r^{2}+\alpha^{2}}\right]^{\mu}}
$$

Proof 1 In essence, it is necessary to show $r^{\mu} \leq\left[\sum_{\alpha=0}^{r} \sqrt{r^{2}+\alpha^{2}}\right]^{\mu}$, in particular for $\mu \geq 2$, expanding the series and factorizing terms, we need to prove

$$
r \leq \sum_{\alpha=0}^{r} \sqrt{r^{2}+\alpha^{2}}=r \sqrt{r\left[\frac{r(r+1)(2 r+1)}{6}\right]}
$$

but, $r(r+1)(2 r+2) / 6=1+2^{2}+3^{2} \ldots+r^{2} \geq 1$ and $r \geq 1$, then

$$
\sqrt{r\left[\frac{r(r+1)(2 r+1)}{6}\right]} \geq 1
$$

therefore is satisfied $r^{\mu} \leq\left[\sum_{\alpha=0}^{r} \sqrt{r^{2}+\alpha^{2}}\right]^{\mu}$ and the Proposition 1 .

Using Proposition 1, it is possible to show

$$
\sum_{r=1}^{\infty} \frac{1}{r^{\mu}} \geq \sum_{r=1}^{\infty} \frac{1}{\left[\sum_{\alpha=0}^{r} \sqrt{\left(r^{2}+\alpha^{2}\right)}\right]^{\mu}},
$$

thus, we can define

$$
z(\mu)=2 \sum_{r=1}^{\infty} \frac{1}{r^{\mu}}=2 \zeta(\mu), \text { and } Z(\mu)=4 \sum_{r=1}^{\infty} \frac{1}{\left[\sum_{\alpha=0}^{r} \sqrt{\left(r^{2}+\alpha^{2}\right)}\right]^{\mu}},
$$

where $\zeta(\mu)$ is the Riemann function and $Z(\mu)$ is a new function adapted to the 2-dimensional case, and is satisfied $\frac{1}{2} Z(\mu) \leq Z(\mu)$ for $\mu>2$, this implies that $Z(\mu)$ is well defined, therefore,

$$
\sum_{j \in \mathcal{Z}^{2}, j \neq i} w_{i \rightarrow j}=\mathcal{L} A_{i}(t)+l^{-\mu} Z(\mu) A_{i}(t)
$$

so, the probability $q_{i \rightarrow d}$ could be expressed in terms of $\mathcal{L}$ and $Z=Z(\mu)$

$$
q_{i \rightarrow d}=\frac{A_{d}(t)}{\|i-d\|^{\mu}}\left(\frac{1}{Z A_{i}(t)}-\frac{\mathcal{L} A_{i}(t) l^{\mu}}{Z^{2} A_{i}^{2}(t)}\right) .
$$

The result of Eq. (11) is used below in Eq. (13). The derivation of the 2-D model, in essence, is the one developed by Chaturapruek [6] for 1-D, which is clearly explained. However, an outline of the deduction is shown below.

The criminal dynamics agents can be highly complex, so limiting the model's scope is required. For this, the following assumptions are proposed:

- At position $i$ for each time interval $\delta t$, two things are possible: (a) Each criminal commits a crime with probability $A_{i} \delta t$, (b) He moves to another location direction that is biased by the attractiveness distribution field.

- New criminals are being created everywhere, with a $\Gamma$ spawn rate. This property allows a regular population of criminals to move continuously to more attractive places.

- House burglars can only move from a site $i$ to a site $d$ by means of $q_{i \rightarrow d}$ or otherwise be generated at $d$, at a rate $\Gamma$.

- A portion $\lambda$, proportional to house burglars $n_{i}(t)$, can cease to operate, leaving without committing a crime.

Expression (12) models the criminal dynamics, established on previous assumptions, such that

$$
n_{d}(t+\delta t)=\sum_{i \in \mathbb{Z}^{2}, i \neq d} n_{i}(t)\left(1-A_{i}(t) \delta t\right) \cdot q_{i \rightarrow d}-\lambda n_{d}(t) \delta t+\Gamma \delta t,
$$


subtracting $n_{d}(t)$ from both sides and dividing everything by $\delta t$, we have

$$
\frac{n_{d}(t+\delta t)-n_{d}(t)}{\delta t}=\frac{1}{\delta t}\left[\sum_{i \in \mathbb{Z}^{2}, i \neq d} n_{i}(t)\left(1-A_{i}(t) \delta t\right) \cdot q_{i \rightarrow d}-n_{d}(t)\right]-\lambda n_{d}(t)+\Gamma
$$

considering only the term into the brackets of the previous equation, and replacing $q_{i \rightarrow d}$ from Eq. (11)

$$
\begin{gathered}
\sum_{i \in \mathbb{Z}^{2}, i \neq d} n_{i}(t)\left(1-A_{i}(t) \delta t\right)\left[\frac{A_{d}(t)}{\|i-d\| \mu}\left(\frac{1}{Z A_{i}(t)}-\frac{\mathcal{L} A_{i}(t) l^{\mu}}{Z^{2} A_{i}(t)^{2}}\right)\right]-n_{d}(t) \\
=A_{d}(t)\left[\left(\sum_{i \in \mathbb{Z}^{2}, i \neq d} \frac{n_{i}(t)}{A_{i}(t)}\left(1-A_{i}(t) \delta t\right) \frac{1}{\|i-d\|^{\mu} Z}\right)-\frac{n_{d}(t)}{A_{d}(t)}\right] \\
\quad-\sum_{i \in \mathbb{Z}^{2}, i \neq d} n_{i}(t)\left(1-A_{i}(t) \delta t\right) \frac{A_{d}(t)}{\|i-d\|^{\mu}} \frac{\mathcal{L} A_{i}(t) l^{\mu}}{Z^{2} A_{i}^{2}(t)}
\end{gathered}
$$

based on the fact that $n_{d}(t)=\sum_{i \in \mathbb{Z}^{2}, i \neq d} \frac{n_{i}(t)}{\|i-d\|^{\mu} Z}$, truncating to order $\mathcal{O}\left(l^{\mu}, \delta t\right)$ and neglecting terms $\mathcal{O}\left(l^{\mu} \delta t, l^{2 \mu}\right)[6]$, we have

$$
\approx A_{d}(t) \sum_{i \in \mathbb{Z}^{2}, i \neq d}\left[\frac{\frac{n_{i}(t)}{A_{i}(t)}-\frac{n_{d}(t)}{A_{d}(t)}}{\|i-d\| \mu Z}-\frac{n_{i}(t)}{\|i-d\| \mu}\left(\frac{\mathcal{L} A_{i}(t) l^{\mu}}{A_{i}^{2}(t) Z^{2}}\right)-\delta t \frac{n_{i}(t)}{\|i-d\| \mu Z}\right],
$$

using the right side of the definition of operator $\mathcal{L}$ in equation (7)

$$
\approx A_{d}(t)\left[\frac{l^{\mu}}{Z} \mathcal{L}\left(\frac{n_{d}(t)}{A_{d}(t)}\right)-n_{d}(t) \frac{\mathcal{L} A_{d}(t) l^{\mu}}{A_{d}^{2}(t) Z}-\delta t n_{d}(t)\right],
$$

substituting the last result (15) in Eq. (13), applying the limit $l, \delta t \rightarrow 0$, and using Eq. (9) that relates the operator $\mathcal{L}$, with $\Delta^{s}$, we have

$$
\frac{n_{d}(t+\delta t)-n_{d}(t)}{\delta t}=\frac{l^{\mu}}{\delta t Z C_{2,2 S}}\left[A(\mathbf{x}, t) \Delta^{S}\left(\frac{n(\mathbf{x}, t)}{A(\mathbf{x}, t)}\right)-\frac{n(\mathbf{x}, t)}{A(\mathbf{x}, t)} \Delta^{S}(A(\mathbf{x}, t))\right]-n(\mathbf{x}, t) A(\mathbf{x}, t)-\lambda n(\mathbf{x}, t)+\Gamma,
$$

in agree to equation (8) for the case $D=2$

$$
C_{2,2 s}=\frac{2^{2 s} \Gamma(s+1)}{\pi|\Gamma(-s)|}
$$

dividing Eq. (16) by $l^{2}$, using the limit $\delta t, l \rightarrow 0$, and the definition $\rho(\mathbf{x}, t)=\lim _{l \rightarrow 0} n_{d}(t) / l^{2}$, we obtain the Lévy Flight Model approximation for criminal density $\rho(\mathbf{x}, t)$, as shown in Eq. (18). On the other hand, from equation (3), is obtained directly Eq. (17) (using $A(\mathbf{x}, t)=$ $\left.B(\mathbf{x}, t)+A^{0}(\mathbf{x})\right)$, as follows

$$
\begin{aligned}
& \frac{\partial A(\mathbf{x}, t)}{\partial t}=\mathrm{\eta} \Delta\left(A(\mathbf{x}, t)-A^{0}(\mathbf{x})\right)-\omega\left(A(\mathbf{x}, t)-A^{0}(\mathbf{x})\right)+\epsilon \rho(\mathbf{x}, t) A(\mathbf{x}, t), \\
& \frac{\partial \rho(\mathbf{x}, t)}{\partial t}=M\left[A(\mathbf{x}, t) \Delta^{S}\left(\frac{\rho(\mathbf{x}, t)}{A(\mathbf{x}, t)}\right)-\frac{\rho(\mathbf{x}, t)}{A(\mathbf{x}, t)} \Delta^{S}(A(\mathbf{x}, t))\right]-A(\mathbf{x}, t) \rho(\mathbf{x}, t)-\lambda \rho(\mathbf{x}, t)+\gamma .
\end{aligned}
$$

With the following definitions:

$$
\eta=\frac{\eta^{*} l^{2}}{4 \delta t}, \quad \epsilon=\epsilon^{*} l^{2}, \quad M=\frac{l^{\mu-2}}{\delta t Z C_{2,2 s}}, \quad \gamma=\frac{\Gamma}{l^{2}}
$$

Numerical integration

A straightforward way to approximate the two-dimensional fractional Laplacian operator is by Fast Fourier Transform (FFT) properties $[26,27]$. The underlying factor that allows us to take advantage of the Fourier transform is that by projecting the fractional Laplacian operator to the Fourier modes space, differential operations are transformed into algebraic operations, which is relatively simple to compute. Subsequently, the inverse transform is applied, thus completing the cycle to approximate the fractional operator. Therefore, the spectral approximation [28] to $\Delta^{S}$ is expressed as follows:

$$
\Delta^{s} A=\operatorname{real}\left\{\mathcal{F}_{2 D}^{-1}\left\{-\left(k x^{2 s}+k y^{2 s}\right) \mathcal{F}_{2 D}\{A\}\right\}\right\}
$$




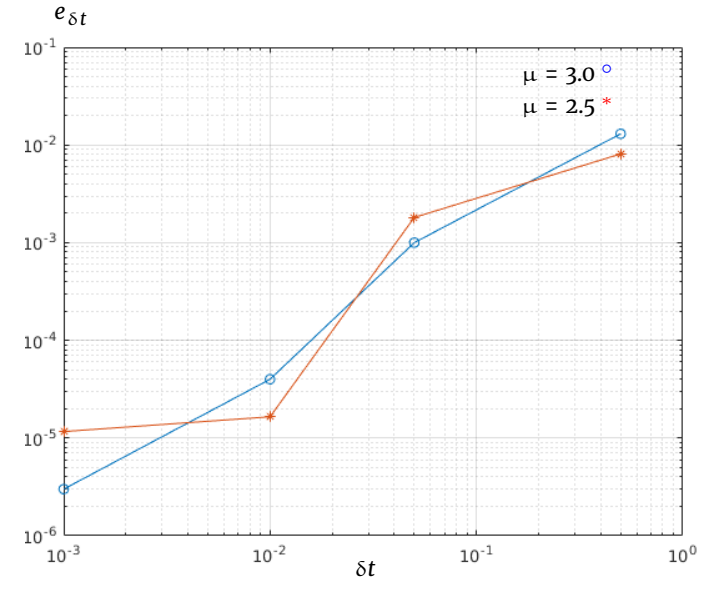

(a)

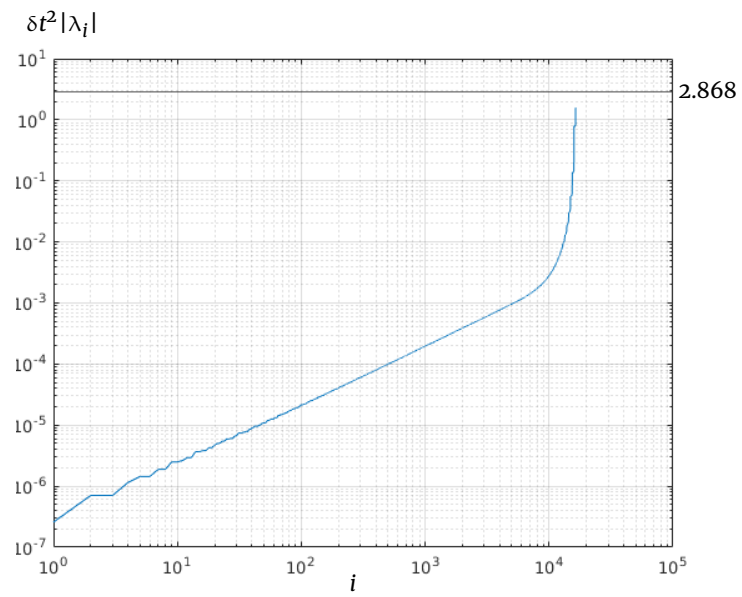

(b)

Figure 1. (a) Relative error approximation $e_{d t}$ as function of $\delta t=\{0.5,0.05,0.01,0.001\}$ after integrating a time $t=200$ using parameters defined in Fig. 2. (b) Values $\delta t^{2}\left|\lambda_{i}\right|$, $i=1 \ldots N^{2}$ of discrete space operator of attractiveness. It can be seen that the stability condition $\delta t^{2}\left|\lambda_{\max }\right|<2.868$ is satisfied, with $\lambda_{\max }=\max \left\{\lambda_{i}\right\}$.

$\mathcal{F}_{2 D}$ represents the Fourier transform in two dimensions, and $k x, k y \in \mathcal{Z}$, are the wavenumbers in each orthogonal direction, respectively. Time derivatives $(\partial A / \partial t, \partial \rho / \partial t)$ are solved via a succession between an explicit and an implicit method, as explained below. Let

$$
f\left(A_{t}, \rho_{t}\right)=\left(\begin{array}{l}
\frac{\partial A(\mathbf{x}, t)}{\partial t} \\
\frac{\partial \rho(\mathbf{x}, t)}{\partial t}
\end{array}\right) .
$$

Step 1: A predictive step is made according to the explicit Adams-Bashforth method [29]

$$
\left.\left(\tilde{A}_{t+\delta t}, \tilde{\rho}_{t+\delta t}\right)=\left(A_{t}, \rho_{t}\right)+\frac{d t}{24}\left[55 f\left(A_{t}, \rho_{t}\right)\right)-59 f\left(A_{t-\delta t}, \rho_{t-\delta t}\right)+37 f\left(A_{t-2 \delta t}, \rho_{t-2 \delta t}\right)-9 f\left(A_{t-3 \delta t}, \rho_{t-3 \delta t}\right)\right] .
$$

Step 2: A correction stage is now implemented, following the implicit Adams-Moulton method

$$
\left(A_{t+\delta t}, \rho_{t+\delta t}\right)=\left(A_{t}, \rho_{t}\right)+\frac{d t}{24}\left[9 f\left(\tilde{A}_{t+\delta t}, \tilde{\rho}_{t+\delta t}\right)+19 f\left(A_{t}, \rho_{t}\right)-5 f\left(A_{t-\delta t}, \rho_{t-\delta t}\right)+f\left(A_{t-2 \delta t}, \rho_{t-2 \delta t}\right)\right]
$$

A successive combination at each time step into explicit (20), and implicit (21) integration procedure, conforms a four-step PredictorCorrector method (PC4) [30]. Three additional steps are generated by applying a fourth-order Runge-Kutta method (RK4) to initiate the integration process. The reason for using PC4 is because of its lower computational cost compared to RK4 [31]. However, exists alternatives, for example, the proposed by C. Tadjeran and M. Meerschaert [32], explicitly designed for fractional operators. To evaluate the convergence of the numerical solution, the definition of relative error $e_{\delta t}=\max \left\{\left[A_{t+\delta t}-A_{t}\right] / A_{t}\right\}$ is used. The calculations of $e_{\delta t}$ with $\delta t=\{0.5,0.05,0.01,0.001\}$ are presented in graph (a) of Fig. 1 for $\mu=\{2.5,3\}$, the rest of parameters are defined in Fig. 2. One method to determine spectral stability in time depending on partial differential equations is to calculate the eigenvalues $\lambda_{i}, i=1 \ldots N^{2}$ of the spatial discretization of the operator $\Delta^{*}=\eta \Delta-\omega+\epsilon \rho(t, \mathbf{x})$ scaled by $\delta t^{2}$ (two dimensions) [28]. Stability condition for PC4 is $\delta t^{2}\left|\lambda_{\max }\right|<720 / 251 \approx 2.868$ [33]. In graph (b) of Fig. 1, the eigenvalues of the discretization of the spatial operator of attractiveness $A$ scaled by $\delta t^{2}$ are shown.

In Fig. 2 initial condition and numerical integration is shown. The parameter values are fixed in $N=256, d t=1 \times 10^{-3}$, integration $t=200$, and $\eta^{*}=3.94, \lambda=0.05, \omega=0.05, \Gamma=0.019, \epsilon=0.10$. (a) Initial condition is an attractiveness random map, the initial burglaries density $\rho(\mathbf{x})_{0}$, is a homogeneous distribution fixed at 0.2 and boundary conditions are periodic. In (b) $\mu=4.40$ corresponds with $s=1.20$. Maximum attractiveness intensity showed an increase compared to the initial condition, achieving a maximum of $\approx 0.8$. (c) $\mu=2.50$ corresponding with derivative order $s=0.25$, in this case, dominate Lévy flights over Gaussian diffusion, it can be seen how attractiveness is concentrated in two principal regions (central hotspots) achieving a maximum of $\approx 1$.6. The non-local effect of fractional operator explains that this plays a relevant role in how the crime field influences attractiveness distribution.

The preceding numerical examples show relatively distant cases compared to the fractional order. The most relevant aspect is the formation of intense few hotspots (central hotspots) in issues where Lévy flights dominate. For example, this result has particular interest for modeling metropolitan areas where attractiveness does not manifest homogeneous space of small hotspots distribution. Still, instead, a few hotspots rise, as is usually the case with city centers, as shown in [21]. In the case of $s \approx 1$ were recovered Laplacian diffusion results.

\section{Approximate analysis for stationary solutions in one dimension}

For some nonlinear phenomena under specific continuity conditions, can be done a study through a power series approximation. This idea is based on the assumption that solutions are analytical functions concerning some of their parameters. The series length is infinite, but an 


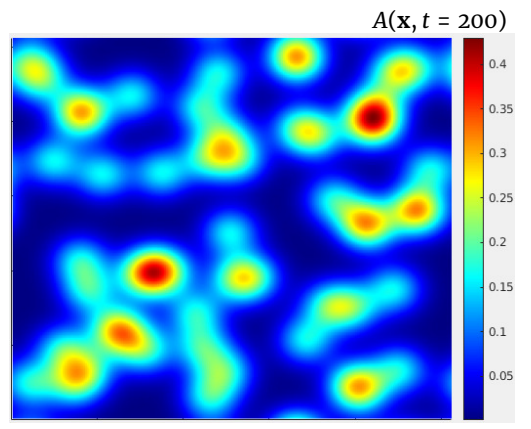

(a) Initial condition

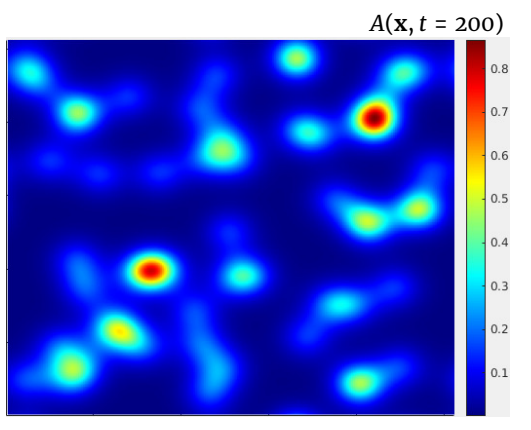

(b) $s=1.20$

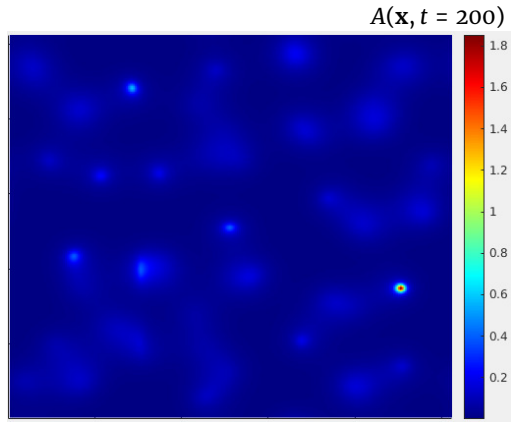

(a) $s=0.25$

Figure 2. (a) Initial condition and (b-c) final state after integrating equations (17), (18) for a time $t=200$. Boundary conditions are periodic for both examples. The parameters are fixed in $N=256, \eta^{*}=3.94, \lambda=0.05, \omega=0.05, \Gamma=0.019$ and $\epsilon=0.10$. Case (b) $\mu=4.40$ corresponding with $s=1.20$, the number of hotspots has been reduced compared to the initial condition, but the magnitude of the intensity has increased. (c) $\mu=2.50$ equivalent to $s=0.25$, in this instance Lévy flights (anomalous diffusion), dominate over Laplacian diffusion, the formation of hotspots with a mayor intensity is observed (respect to (b)). These can be explained as the non-spatial locality effect of the fractional operator, which allows the crime density field $\rho$ to have a more significant influence on the appearance of a few high-intensity hotspots in the attractiveness $A$.

approximate model is obtained by truncating to a specific power order. The precision radius is usually a function of the power order trimmed in the series. Expanding series analysis is applied to estimate the local phenomenology of the original system through an approximate model. There are studies using series expansion on stationary solutions for the Short model $[2,7,10]$. Other studies have also been made to assess their stability $[3,8,15,34]$ and bifurcation analysis [35, 36]. Our study proposes a series expansion analysis, considering the density home burglaries field as an analytical function of attractiveness. A comparable analysis is undertaken in reference [6], although the ansatz for expansion is different from the one suggested here, as shown below. Using equation (17) in the steady-state i.e. $\partial A / \partial t=0$, also, consider special case $A^{0}=\tilde{\alpha}$ (constant in all $\Omega$ ), we propose

$$
\eta \Delta A-\omega A+\epsilon A \rho(A)+\omega \tilde{\alpha}=0, \text { and } \rho(A)=\sum_{i=0}^{\infty} \tilde{\beta}_{i}(s) A^{i} .
$$

Replacing $\rho(A)$, dividing by $\eta$, and ordering by powers of $A$, we have

$$
\Delta A-\frac{\left(\omega-\epsilon \tilde{\beta}_{0}\right)}{\eta} A+\frac{\epsilon \tilde{\beta}_{1}}{\eta} A^{2}+\frac{\epsilon \tilde{\beta}_{2}}{\eta} A^{3}+\ldots=-\frac{\omega \tilde{\alpha}}{\eta},
$$

renaming the coefficients, as $\beta_{1}=\frac{\left(\omega-\epsilon \tilde{\beta}_{0}\right)}{\eta}$, for $i \geq 2 \beta_{i}=\frac{\epsilon \tilde{\beta}_{i-1}}{\eta}$ and $\alpha=\frac{\omega \tilde{\alpha}}{\eta}$, therefore,

$$
\Delta A-\beta_{1}(s) A+\beta_{2}(s) A^{2}+\beta_{3}(s) A^{3}+\ldots=-\alpha .
$$

For qualitative analysis, we will work on one dimension $\left(\Delta A=A_{x x}\right)$, and the last expression is truncated to the third power in $A$. Which provides us with a non-homogeneous and non-linear ordinary differential equation

$$
A_{x x}-\beta_{1}(s) A+\beta_{2}(s) A^{2}+\beta_{3}(s) A^{3}=-\alpha .
$$

For the analysis, we propose changing the second-order differential equation (23) for two first-order equations, as follows:

$$
\begin{aligned}
& A_{x}=M, \\
& M_{\chi}=\beta_{1}(s) A-\beta_{2}(s) A^{2}-\beta_{3} A^{3}-\alpha .
\end{aligned}
$$

One further approximation is required to introduce the functional relationship between $\beta_{i=1,2,3}$, with respect to the derivative order $s$. In a small disturbances scheme (concerning intrinsic attractiveness), a linear relationship is suggested for the three functions, such that $\beta_{i}(s)=a_{i}$ s. Fig. 3 presents the numerical solution for this approximation level. In Fig. 3 (a) the configuration space $M(\mathbf{x})$ vs $A(\mathbf{x})$ is plotted for several values of derivative order $s \in[0.2,1.4]$. In Fig. 3 (b) Solution of eqs. (24) is shown with coefficients $a_{1}=100, a_{2}=8, a_{3}=8$, which resemble spike solutions. (c) For the complete model (eqs. (17)-(18) in 1-Dimension) is shown $M(\mathbf{x})$ vs $A(\mathbf{x})$, with parameters $\eta=3.874, \omega=0.05, \lambda=0.09, \Gamma=5 \times 10^{-6}, \epsilon=0.04, s \in[0.2,1.4]$, the initial conditions are $A(\mathbf{x}, 0)=(1-\cos (2 \pi \mathbf{x})), \rho(\mathbf{x}, 0)=0.1$ and the subjacent attractiveness $\alpha=A^{0}(\mathbf{x})=0.1$. (d) Are shown solutions of $A(\mathbf{x}, t=200)$, for different derivative order values $s \in[0.2,1.4]$. In sub Figs. (a)-(d), curves with the maximum amplitude correspond with lower values of $s$, and the amplitude decrease when $s$ is incremented. Visually, it can be seen that the behavior of the real and approximate solutions are qualitatively different. By tuning the parameters in the approximate model, the amplitude and width of the curves can be adjusted, however, to modify the shape of the curve a different model is required. With this observation, we can say that an approximation by a few terms is not enough to capture the dynamic of the attractiveness represented by Eq. (17). Also, the linear relationship between the coefficients $\beta_{i=1,2,3}$ and the order of the derivative $s$, seems not to be adequate to approximate the behavior of the system. 


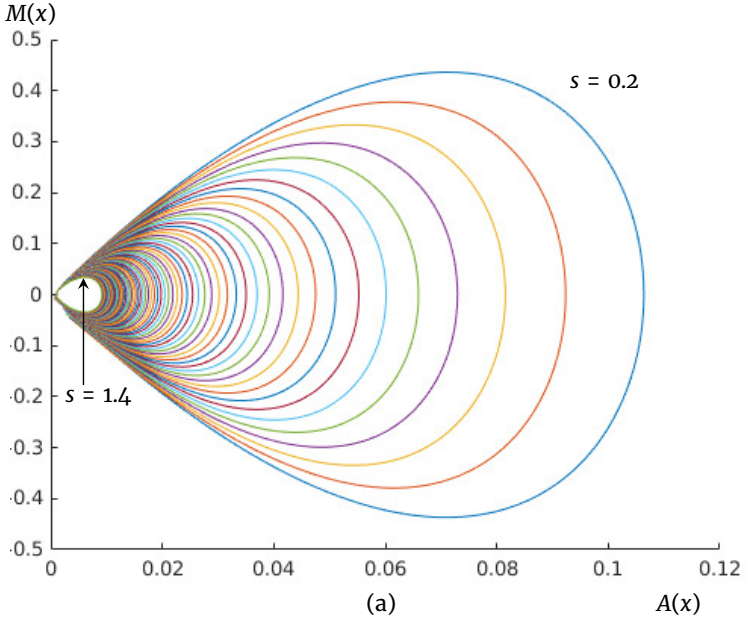

(a)

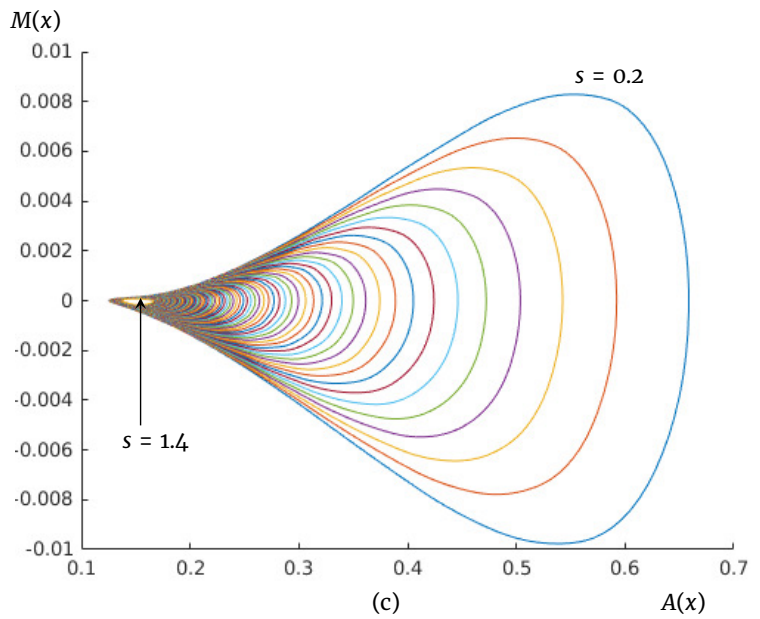

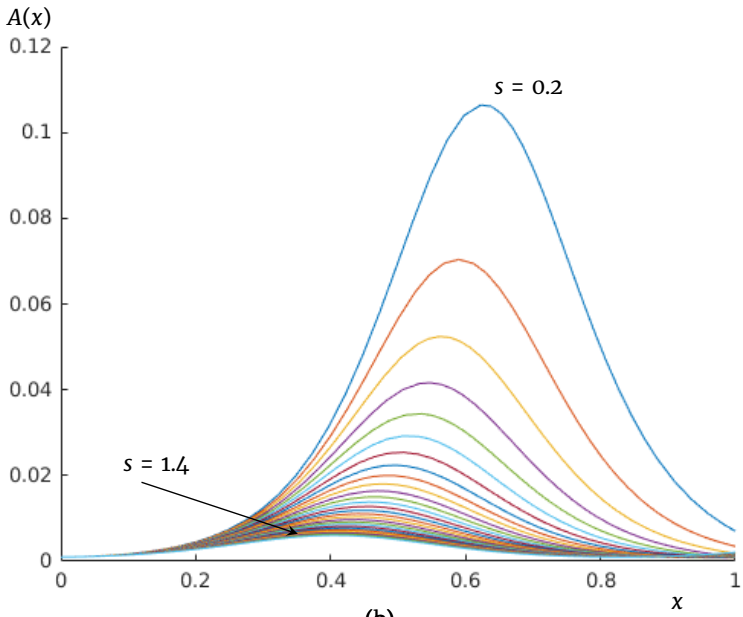

(b)

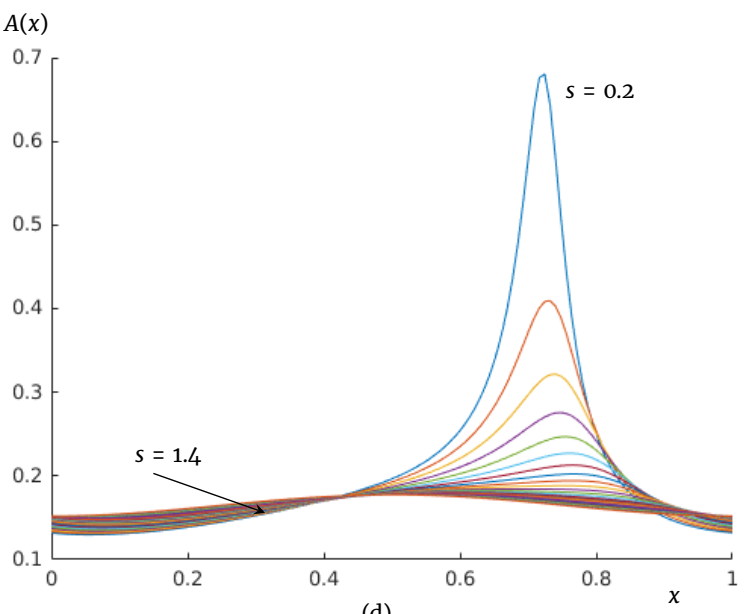

(d)

Figure 3. Graphical comparison between complete (eqs. (17) and (18) in 1-dimension) and approximated model (Eq. (24)). (a) Evolution of different derivative order values $s \in[0.2,1.4]$ for $M(x)=\partial A(x) / \partial x$ vs $A(x)$. (b) Spatial distribution of $A(x)$ for $s \in[0.2,1.4]$. (c) Solution of the complete model (eqs. (17), (18) in 1-dimension) for derivative order $s \in[0.2,1.4]$. (d) Spatial distribution of the solution $A(\mathbf{x}, t=200)$ for $s \in[0.2,1.4]$. The parameters of the approximation $($ Eq. $(24))$ are $\beta_{i}(s)=a_{i} s$ with $a_{1}=100, a_{2}=8$, $a_{3}=8$. Parameters of the eqs. (17) and (18) are $\eta=3.874, \omega=0.05, \lambda=0.09, \Gamma=5 \times 10^{-6}, \epsilon=0.04$ and $\alpha=A^{0}(\mathbf{x})=0.1$. It is appreciated that an approximation of a few terms and linear relations of the $\beta_{i=1,2,3}$ coefficients and $s$ does not consistently capture the dynamics of the complete model. However, the relation between curve amplitude and fractional derivative is represented in a qualitative way. 


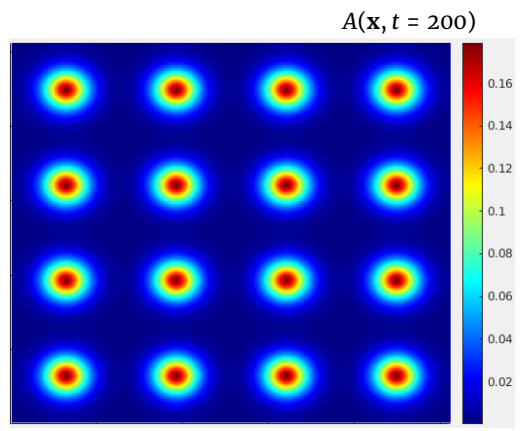

(a) $s=0.17$

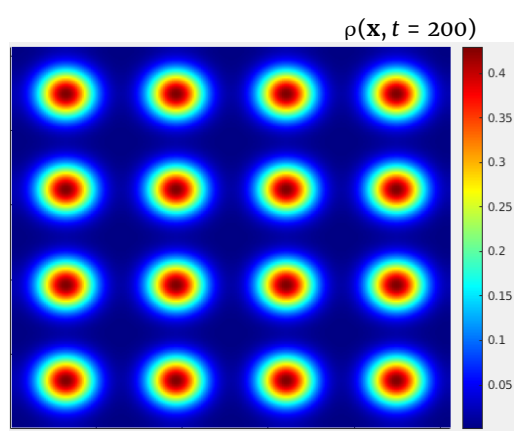

$(d) s=0.17$

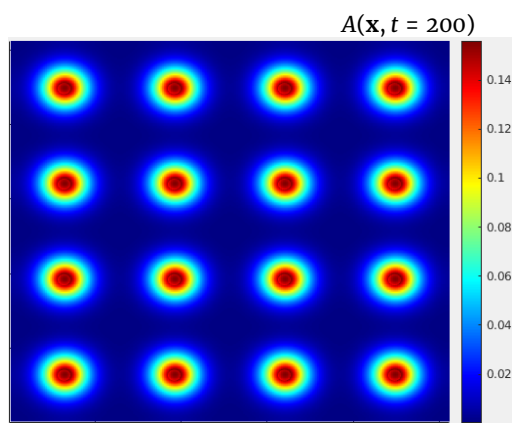

(b) $s=0.25$

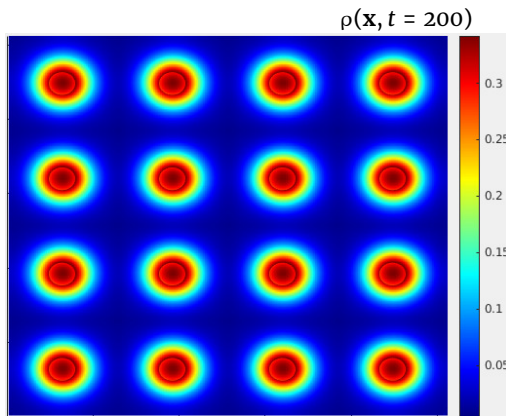

$(e) s=0.25$

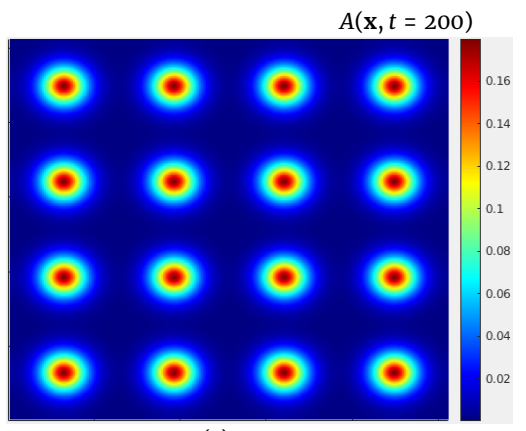

$(c) s=0.29$

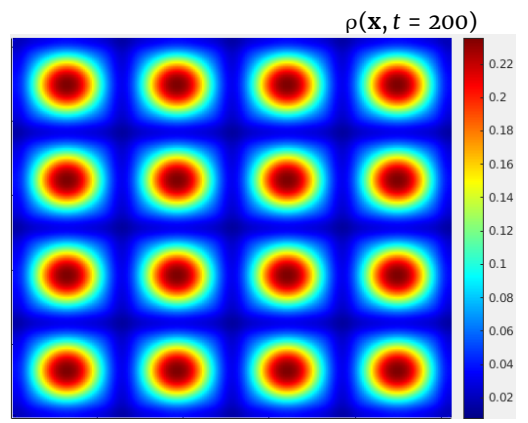

$(f) s=0.29$

Figure 4. Numerical attractiveness solution $A(x, t)$, after integrating for $t=200$. (a), (b) and (c) represent the attractiveness field for $s=\{0.17,0.25,0.29\}$ respectively. Figures (d), (e) and (f) correspond to the criminal density distribution field for (a), (b) and (c) respectively. The three scenarios show in the attractiveness field a hotspot's existence coupled spacially with hotspots in the criminal density field. For case (a)-(d), the attractiveness hotspots have a localized and intense dot at the center, and the criminal density field exhibits similar behavior. In (b)-(d) appears an intense ring around the center of the hotspot. Those phenomena occur in attractiveness and criminal density fields. In figures (c) -(f), the attractiveness increases marginally concerning the previous cases. The hotspots in crime density are less intense than (a)-(c) and (b)-(e).

\section{Numerical analysis of fractional order in 2-dimensions}

Using the integration method explained in Subsection 2, several bi-dimensional scenarios were calculated, while varying s. The initial condition $A_{0}(\mathbf{x})$ for all cases is composed of an array with $4 \times 4$ Gaussian distributions on the domain $\Omega=[0,1] \times[0,1]$ and periodic boundary conditions. In Fig. 4 , the final state is shown after integrating by $t=200$ for $s=\{0.19,0.25,0.29\}$, with parameters $\eta=3.947^{*}$, $\omega=0.05, \lambda=0.05, \Gamma=0.0019, \epsilon=0.10$. As observed in Figs (a)-(c) (attractiveness) and (d)-(f) (density criminal agents), exist different behavior regimes for values of $s<0.45$, which is where the Lévy flights dominate over conventional diffusion. Although there are not quite significant changes in the attractiveness hotspots magnitude in (a)-(c), there are more noticeable changes in criminal agents density distribution (d)-(f). To explain this change is necessary to understand criminal density dynamics, as we hypothesized below. In the space of criminal density, Figs. (d)-(f) a descending difference in the magnitude of the hotspot can be observed, while sincreases, simultaneously the hotspot base becomes wider. The hypothesis is that stochastic flights are longer and directed to the most attractive areas, with greater precision, while $s<1$. When derivative order $s$ increases, Lévy flights exist, but now they compete with Laplacian diffusion.

In agreement with numerical observations (Fig. 4), there are two-dimensional periodic solutions for different derivative order values s. However, there are considerable differences between them, analogous to the one-dimensional case reported by Chaturapruek et al. [6]. The magnitude and spatial distribution of the attractiveness field and the criminal density constitute most of these differences. The results interpretation is that for $s<1$, the fractional operator $\Delta^{s}$ manifests its non-local nature. From graphs of Fig. 4, the maximum attractiveness intensity variation is observed by changing parameter $s$. The two-dimensional system solutions are determined numerically for $s \in[0.17,1.20]$. To analyse results, we determine global properties $\max [A] / \max \left[A_{0}\right]$ (max for all $\mathbf{x}$ on $\Omega$ ), as a function of $s$.

In Fig. 5 it is observed how the total attractiveness $\mathrm{I}\left[A_{S}\right]$, defined in equation (25) shifts with respect to the total attractiveness of the initial condition I $\left[A_{0}\right]$. In graphs (a) and (b) of Fig. 5, both properties are shown, as well as the integration time $t=200$. Graph (a) for $s<0.5$ corresponds to a regime where Lévy flights dominate Laplacian diffusion. The Maximum attractiveness reaches high values compared to the rest of the graph. The region $0.5<s$ shows $\max A_{s} / \max A_{0}$ has small variations, but it shows a local maximum at $s \approx 1$. In graphs (a)-(b), it is observed that in the case $s>0.5$, the attractiveness magnitude remains low (respect to the case $s<0.5$ ) and continues with this trend, for $s$ explored in this experiment. The hypothesis to explain these two regimens is a behavior change between them from Lévy flights diffusion to one where the Laplacian diffusion has relevant effects or dominates. As a result of the analysis of graphs, it can be said that in an environment where criminal agents have high mobility, attractiveness increases significantly for usual Laplacian diffusion.

$$
\mathrm{I}\left[A_{s}\right]=\int_{\Omega} A_{S}\left(\mathbf{x}, t=T_{C}\right) d \mathbf{x} \text { and } \mathrm{I}\left[A_{0}\right]=\int_{\Omega} A_{0}(\mathbf{x}) d \mathbf{x}
$$

In Fig. 5 the substantial increase in both global attractiveness properties, for values of $s<0.45$ reveals a significant criminal population is leaving its neighborhood and is continually moving to the most attractive areas. This mechanism is reinforced by a cyclical process and 

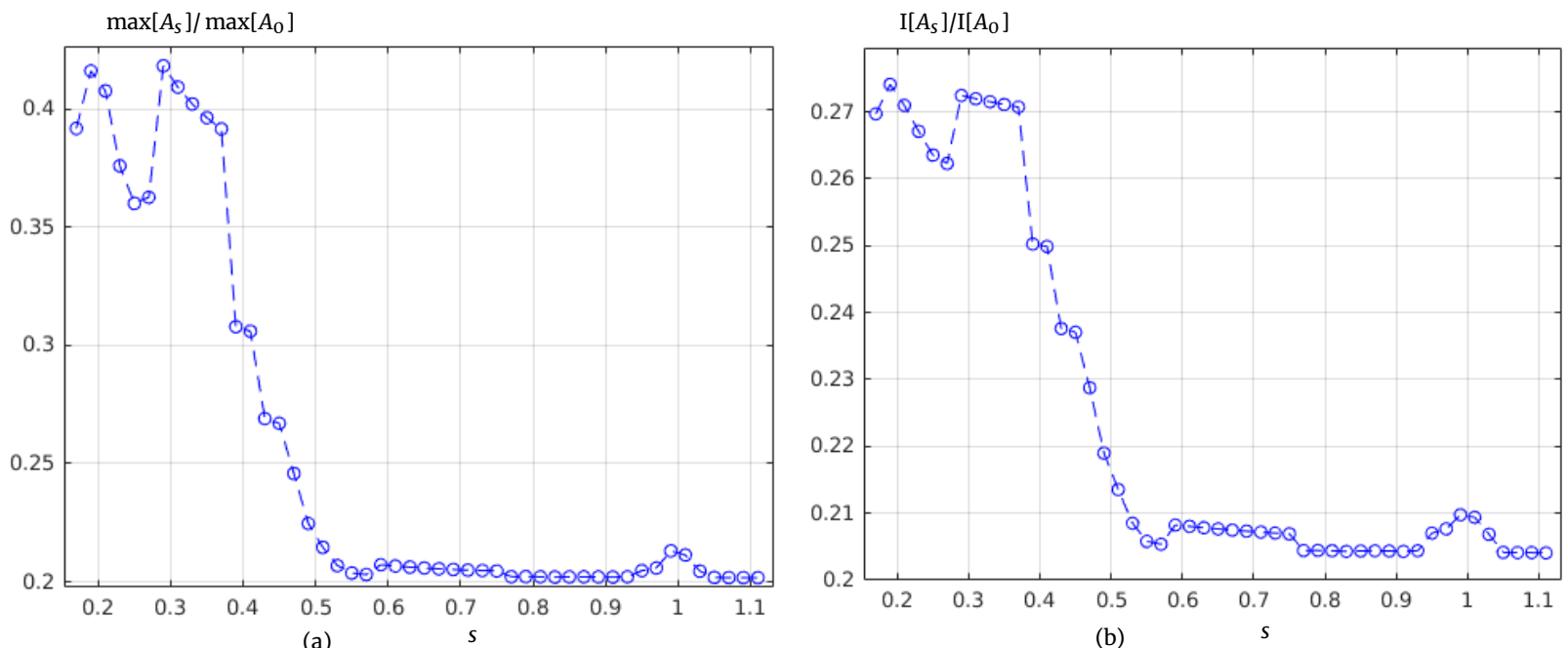

Figure 5. In both graphs the parameters are fixed at $T_{c}=200, \eta=3.947, \omega=0.05, \lambda=0.05, \Gamma=0.0019, \epsilon=0.10$ and $s \in[0.17,1.20]$. (a) Evolution of $\max \left[A_{s}\right] / \max \left[A_{0}\right]$, which measures the maximum attractiveness values in $\Omega$. Graph (b) shows $\mathrm{I}\left[A_{s}\right] / \mathrm{I}\left[A_{0}\right]$, which represents a total attractiveness measurement in $\Omega$. As the previous graph, this shows two attractiveness regimes with a transition zone between them, for the same values of $\Omega$, although, there is a significant decrease when $s=1$, which corresponds to the usual Laplacian diffusion. In both graphs, there is evidently a region for values of $s<0.4$ where essentially there is great attractiveness, both at the maximum intensity level, as in case (a), and global attractiveness, as in case (b). The explanation for this increase in attractiveness relates to greater mobility among the criminal population.

is only limited by local diffusion. In fact, for $s \lesssim 0.37$, the numerical solutions show a remarkable increase, as local criminal diffusion is not enough to delocalize the high attractiveness concentration, and the integration process is numerically unstable. Another exciting aspect is the apparition of a local maximum for $s \approx 1$ because evidence of a substantial change occurs within the usual Laplacian diffusion regime (for the case $s=1$, the conventional definition of the Laplacian operator is used) in this regime, an intense local diffusion increase the attractiveness. In Fig. 5 (b) is shown the total attractiveness on $\Omega$, it has a similar trend to that of the graph (a), that is, the global attractiveness also changes as a function of $s$.

\section{Law enforcement of fractional bi-dimensional model}

How police officers engage with the attractiveness field is fundamental to the model dynamics. As mentioned before, there are several law enforcement strategies for Laplacian diffusion, and each one produces different results $[4,9,11]$, particularly in the work of $\mathrm{N}$. Rodriguez [37], a complete study is made of different patterns on hotspot policing. The usual Laplacian diffusion models primarily represent these, and those fractional models represent only one dimension. Two main components maintain the incorporation of police officers: (a) Displacement dynamics over the environment, i.e., displacement rules. (b) The way criminals interact with the environment; what makes criminals perceive particular sites as less attractive. The strategy applied in this work is cops on the dots, considering this as a typical law enforcement example. Therefore, police agents' existence modifies criminal attractiveness perception in the following way

$$
\tilde{A}_{d}(t)=e^{-\chi k_{d}(t)} A_{d}(t)
$$

where $\chi>0$ represents police influence on criminal perception. The probability that a criminal agent performs an attack on the site $d \in \Omega$ at time $t+\delta t$ is expressed as

$$
\tilde{p}_{d}(t)=\frac{\epsilon \tilde{A}_{d}(t)}{1+\epsilon \tilde{A}_{d}(t)} .
$$

A similar deduction to that described by the equations (1) and (3), is made, for attractiveness in the police presence $A(\mathbf{x}, t)$, and residential burglaries $\rho(\mathbf{x}, t)$. In the continuous limit, we have

$$
\frac{\partial A(\mathbf{x}, t)}{\partial t}=\eta \Delta A(\mathbf{x}, t)-\omega\left(A(\mathbf{x}, t)-A_{0}\right)+\epsilon \theta \tilde{A}(\mathbf{x}, t) \rho(\mathbf{x}, t) .
$$

In the case of $\rho(\mathbf{x}, t)$, the attractiveness change, expressed in equation (26), is exhibited in a variety of probability $q_{i, d}$ to go from a site $i$ to $d$ in a $(t, t+\delta t)$ period, as follows:

$$
q_{i \rightarrow d}=\frac{w_{i \rightarrow d}}{\sum_{d \in \mathcal{Z}, d \neq i} w_{i \rightarrow d}},
$$

where the weight $w_{i \rightarrow d}$ is defined as

$$
w_{i \rightarrow d}=\frac{\tilde{A}_{d}(t)}{|\mu||i-d| \mid \mu},
$$


and the sum of the weights is

$$
\sum_{d \in \mathcal{Z}^{2}, d \neq i} w_{i \rightarrow d}=\sum_{d \in \mathcal{Z}^{2}, d \neq i} \frac{\tilde{A}_{d}(t)-\tilde{A}_{i}(t)}{\left.|\mu||i-d|\right|^{\mu}}+\sum_{d \in \mathcal{Z}^{2}, d \neq i} \frac{\tilde{A}_{i}(t)}{|\mu||i-d|^{\mu}} .
$$

Using equation (9) for the operator $\mathcal{L}$, and with a similar deduction to that shown in section 2, then we have:

$$
\frac{\partial \rho(\mathbf{x}, t)}{\partial t}=D\left[\tilde{A}(\mathbf{x}, t) \Delta^{S}\left(\frac{\rho(\mathbf{x}, t)}{A(\mathbf{x}, t)}\right)-\frac{\rho(\mathbf{x}, t)}{\tilde{A}(\mathbf{x}, t)} \Delta^{S}(\tilde{A}(\mathbf{x}, t))\right]-\tilde{A}(\mathbf{x}, t) \rho(\mathbf{x}, t)+\gamma .
$$

Values for $D$ and $\gamma$ are specified in equation (19). A relevant aspect of the cops on the dots strategy is that those police officers are biased towards the most attractive areas. These phenomena generate a masking effect that reduces the attractiveness perception of criminals concerning specific places, thus forcing them to move to less attractive areas or disappear from the scene without ever committing a crime. The probability that a police officer will move from site $i$ to site $d$ is

$$
q_{i \rightarrow d}(t)=\frac{A_{d}(t)}{\sum_{i \sim d} A_{i}(t)}
$$

the expected number $k$ of police agents at site $d$ over time $t+\delta t$ is expressed as

$$
k_{d}(t+\delta t)=\sum k_{i}(t) q_{i \rightarrow d}(t)
$$

Chaohao [11] make a similar deduction for the continuous limit, which, if adapted to the two-dimensional case, can be expressed as:

$$
\frac{\partial k}{\partial t}=\tilde{D} \nabla \cdot\left[\nabla k-\frac{2 k}{A} \nabla A\right]
$$

where $\tilde{D}=\omega D$. The equations (27)-(29) represent the fractional diffusion model with law enforcement, which will be used for the simulations below. The model parameters were set in $T_{C}=200, \eta^{*}=3.94, \lambda=0.05, \omega=0.05, \Gamma=0.019, \epsilon=0.15, s \in[0.17,1.20]$ and the effect of the law enforcement $\chi=0.0,0.86,3.86$. Similar to the section 4 , the maximum and the attractiveness total sum over $\Omega$, is determined integrating and divided by the maximum and the total sum, respectively, of the initial condition $A_{0}$.

A relevant aspect to the law enforcement incorporate, then integration of the system (Eqs. (27)-(29)) becomes unstable for $s<0.5$, although it also depends on the value of $x$. This phenomenon is notorious because the system shows a large ring formation around the hotspot center before blowing up.

In Fig. 6 (a) the $\max \left[A_{s}\right] / \max \left[A_{0}\right]$ for $\chi=0.0,0.86,3.86$ indicated with blue, green and red colors respectively, are shown. The missing points correspond to the cases with law enforcement $\chi=0.96,3.86$ and, it is where the model could not be integrated numerically for a time $t=200$ as observed for values $s<0.45$, the maximum attractiveness magnitude, grows with the police presence, at least for case $x=0.86$, i.e., attenuation in the attractiveness by law enforcement was expected, but in a nonintuitive response of the system, it was increased $(s<0.45)$. Reading this result is not easy and possibly not unique, but one interpretation is that criminal agents move relatively easily to hotspots, while police officers move by Laplacian diffusion and therefore are slower. Thus, the police agents slowly concentrate on some hotspots, but the criminals can create new hot zones without allowing the police agents to react adequately. For values $s>0.45$, the maximum attractiveness is reduced by the police presence, which is an expected result. Fig. (b), representing the total attractiveness in $\Omega$, shows similar behavior to Fig. (a). A rapid criminal diffusion based on Lévy flights, and a slow police response, have increased global attractiveness levels. It can be concluded that both graphs in Fig. 6 show two types of attractiveness response to law enforcement: First, for a certain intensity of Lévy flights, the attractiveness increases with the law enforcement, and second, the attractiveness is attenuated when the criminal diffusion is comparable to the police diffusion. It shows that different surveillance strategies should be evaluated to find an effective response of police agents to avoid criminal attacks.

Fig. 7 (a)-(b) presents stable attractiveness results $A(x, t=200)$, for the values of $s=0.31$ with $x=0.86$ and $s=0.45$ with $x=3.86$ respectively. These results correspond to the first points on the left of the graphs in Fig. 6 with $\chi>0$. In (a), a ring with less angular symmetry is shown, and the intensity of attractiveness drops suddenly for a critical radius. In addition, isolated spots are observed inside the ring. In numerical tests for values of $s<0.31$, these isolated points grow without limit. The attractiveness dynamic resulting in the spatial distribution observed in (a) is difficult to explain. However, a hypothesis is that a weak police presence in an environment of high criminal mobility fragments usual hotspots into a more complex structure. In Fig. (b), conventional hotspots are observed, the deterrent police influence can be appreciated too, in the attractiveness magnitude. However, a symmetry break has occurred. The upper right corner hotspot began to grow more than the rest. For $s<0.45$ and $x=3.86$, structures similar to case (a) appear, but they blow up before the integration time reaches $t=200$. 

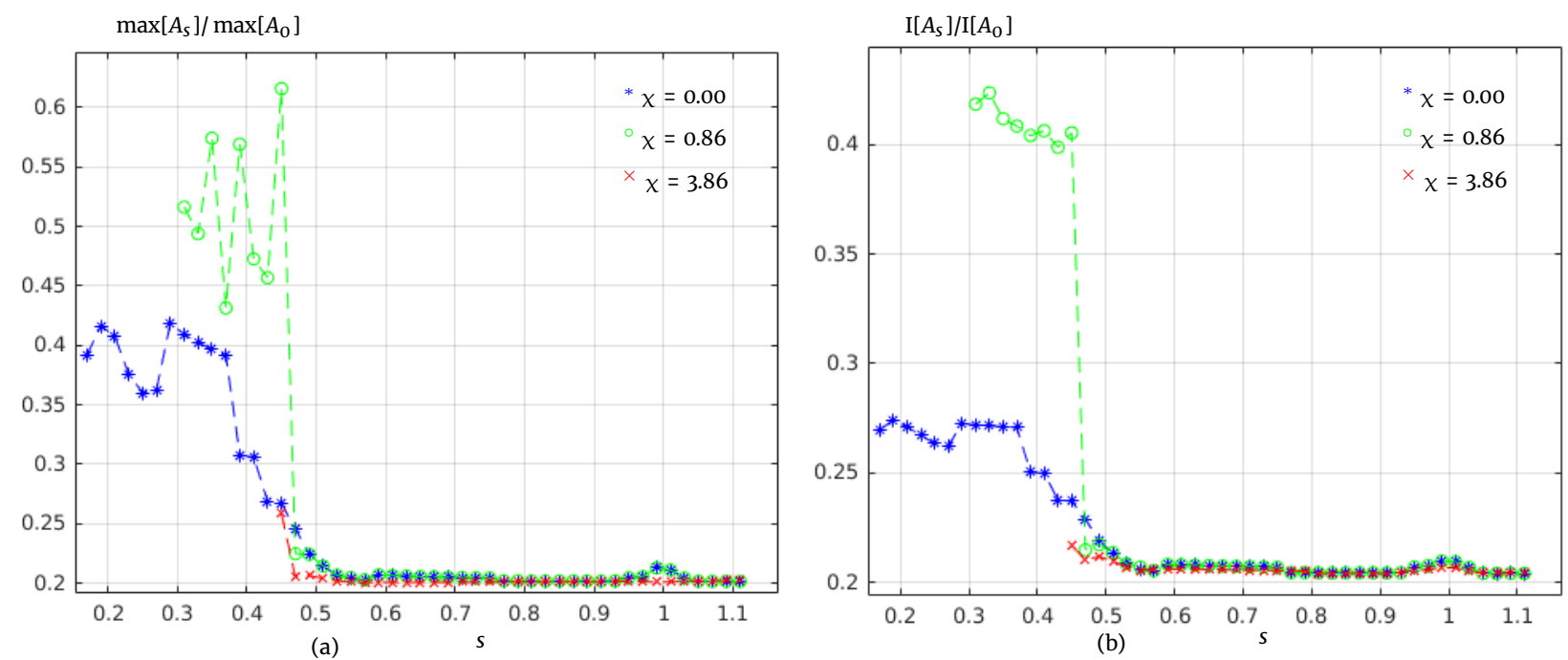

Figure 6. In both graphs the parameters are fixed in $T_{C}=200, \eta=3.94, \lambda=0.05, \omega=0.05, \Gamma=0.019, \epsilon=0.15, s \in[0.17,1.20]$. (a) Evolution of max $\left[A_{s}\right] / \max \left[A_{0}\right]\left(A_{0}\right.$ is the initial condition), Which is a measure of the maximum attractiveness values in $\Omega$, for $\chi=0.0,0.86,3.86$. Graph (b) shows $\mathrm{I}\left[A_{s}\right] / \mathrm{I}\left[A_{0}\right]$, which represents a measure of total attractiveness in $\Omega$ to $x=0.0,0.86,3.86$. Like the previous graph, the existence of two attractiveness regimes exists, the first $(s<0.45)$ is dominated by Lévy flights, and the second $(s>0.45)$ corresponds to a more conventional diffusion.

$A(\mathbf{x}, t)$

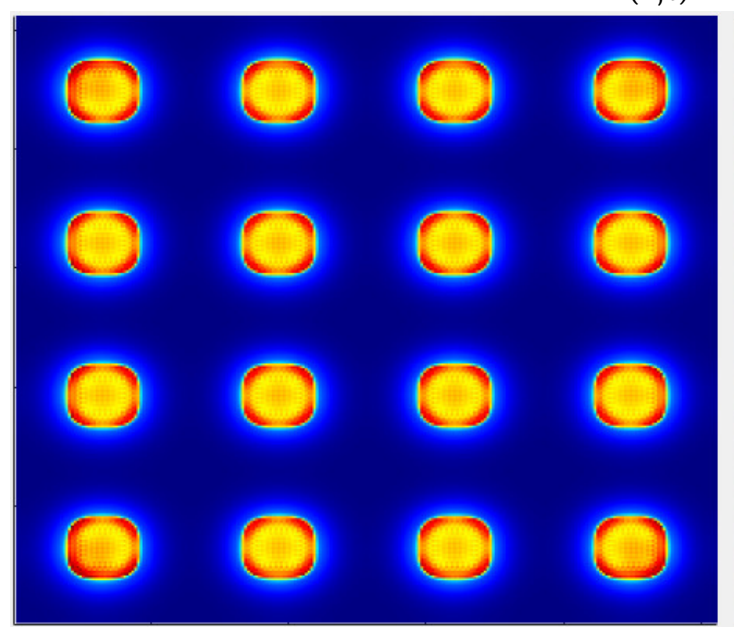

(a)
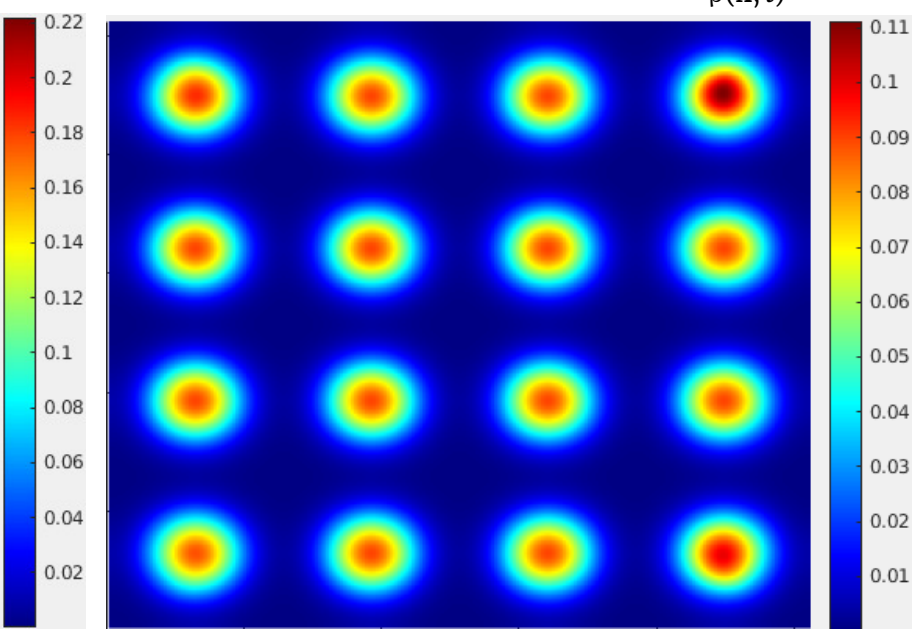

(b)

Figure 7. Spatial distribution for the $\chi=3.86$ and $s=0.31$ cases, the rest of the parameters are those specified in Fig. 6 . (a) Field of attractiveness: A type of hotspot with a shape that varies from the classic one (circular) is visible. (b) The result of the integration is a more conventional hotspot distribution, however, some hotspots have started to grow more than others. 


\section{Conclusions}

We have made a numerical study of the fractional model for the bi-dimensional case of home thieves dynamics, incorporating the police effect and using the Cops on dots strategy. The fractional operator was approximated through two-dimensional Fourier transform properties. It is observed that applying a Predictor-Corrector 4 schedule to a random initial attractiveness distribution, the number and hotspot magnitude are related to the derivative order $s$ of the fractional operator. In a 1-dimensional scenario, stationary solutions analysis found that a series expansion and linear relations between series coefficients with derivative order are not adequate to approximate the functional relationship between attractiveness and criminal density population, inclusive for the small attractiveness amplitudes. A global properties analysis is made, the maximum and total attractiveness were used as estimators of the system evolution. The results from 2-dimensional scenarios reveal two regions with highly contrasting attractiveness behavior. The hypothesis is that Lévy flights dominate the powerful attractiveness region when derivative order ( $s<0.45$ super diffusive regimen). In the interval $s \in[0.45,0.5]$, the dynamics combine the impact of Lévy flights with Laplacian diffusion. An appreciable variation emerges due to a smooth change in global maximums attractiveness for $s=1$; It is explained by the functional form of the coefficient $D_{s}$. Still, the interpretation is that the local diffusion of criminal agents is based entirely on Brownian motion and produces its maximum effect on attractiveness. Incorporating dissuasive police effect into the model, maximums and total attractiveness are significantly reduced for $s>0.5$. In case $s<0.5$, which corresponds to a regimen where Lévy flights coexist or dominate, it is observed that surveillance type cops on the dots increase attractiveness levels in localized areas, with a more complex structure than hotspots. Also, it is interesting that the police presence induces an abrupt change in the system's evolution concerning the derivative order $(s<0.5)$. Numerical results with police influence showed an attractiveness distribution with a different symmetry than the classical hotspots with circular symmetry. However, a more detailed study is required to determine its dynamical properties. Extending the fractional model to the 2-dimensional case brings us closer to a possible application in realistic urban environments, implementing an optimal control investigation. Furthermore, it can be adequate for government agencies to identify attractive home zones and implement optimal surveillance strategies.

\section{Nomenclature}

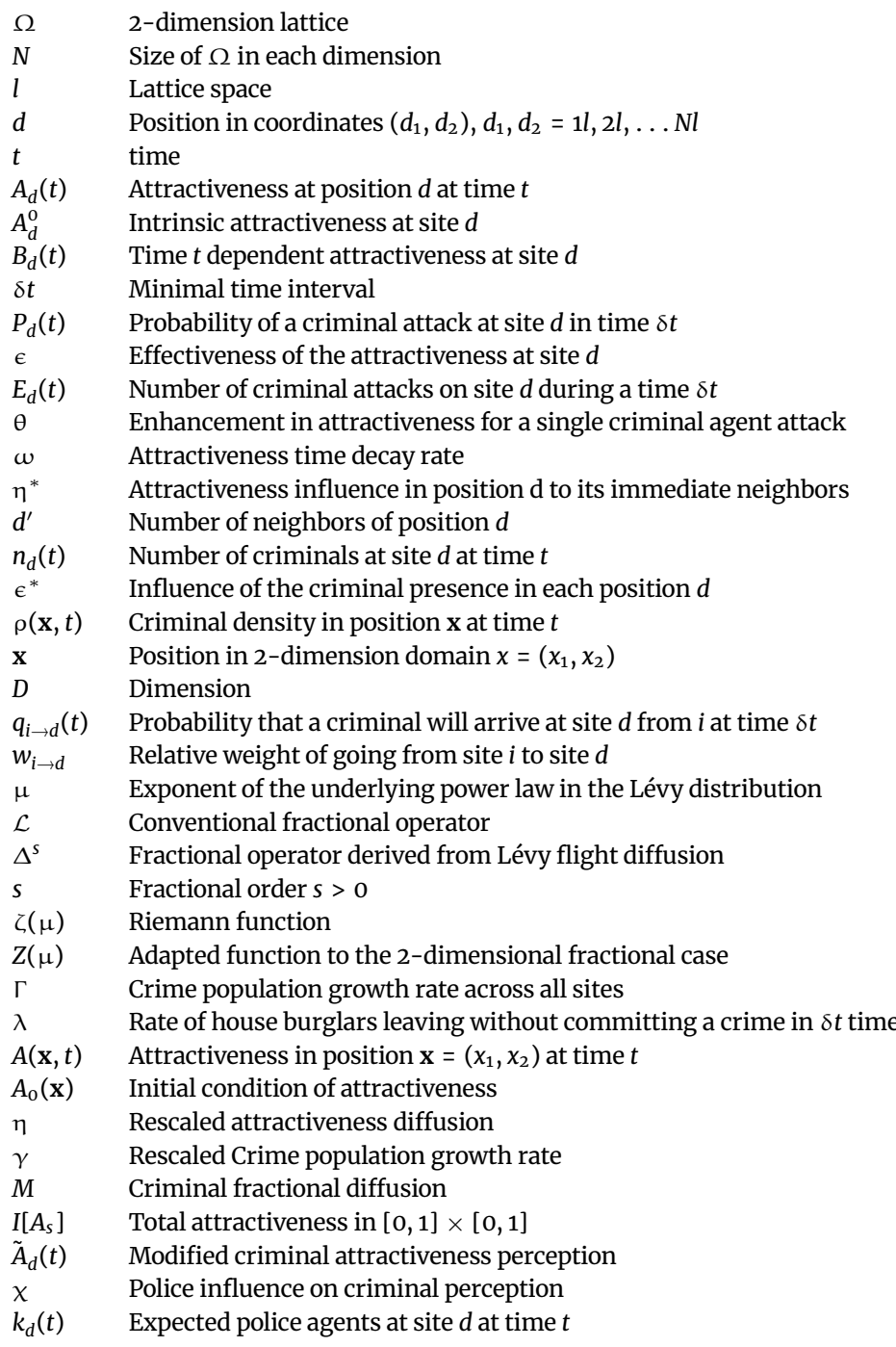




\section{Declarations}

\section{Consent for publication}

Not applicable.

\section{Conflicts of interest}

The authors declare that they have no conflict of interests.

\section{Funding}

Not applicable.

\section{Author's contributions}

F.J.M-F.: Conceptualization, Methodology, Investigation, Writing-Original draft. A.A-S.: Software, Methodology, Data Curation, WritingOriginal draft. R.R-C.: Visualization, Investigation, Software, Writing-Reviewing and Editing. A.H-H.: Methodology, Supervision. All authors discussed the results and contributed to the final manuscript.

\section{Acknowledgements}

F. J. Martínez-Farías was partially supported by the grant UAEHPTC764, DSA/5116/178021. We would like to thank the Supercomputing Laboratory of Apan Research Group "Energy Systems and Advanced Materials" for providing us access to supercomputing resources.

\section{References}

[1] Brantingham, P. \& Tita, G. Offender Mobility and crime pattern formation from first principles. (In L. Liu, \& J. Eck (Ed.), Artificial Crime Analysis Systems: Using Computer Simulations, 2008). [CrossRef]

[2] Short, M., D'Orsogna, M., Pasour, V., Tita, G., Brantingham, P., Bertozzi, A. \& Chayes, L. A Statistical Model of Criminal Behavior Mathematical Models And Methods In Applied Sciences, 18, 1249-1267, (2008). [CrossRef]

[3] Short, M.B., Bertozzi, A.L., \& Brantingham, P.J. Nonlinear patterns in urban crime: Hotspots, bifurcations, and suppression. SIAM Journal on Applied Dynamical Systems, 9(2), 462-483, (2010). [CrossRef]

[4] Jones, P.A., Brantingham, P.J., \& Chayes, L.R. Statistical models of criminal behavior: the effects of law enforcement actions. Mathematical Models and Methods in Applied Sciences, 20(supp01), 1397-1423, (2010). [CrossRef]

[5] Berestycki, H. \& Nadal, J.P. Self-organised critical hot spots of criminal activity. European Journal Of Applied Mathematics, 21(4-5) 371-399, (2010). [CrossRef]

[6] Chaturapruek, S., Breslau, J., Yazdi, D., Kolokolnikov, T. \& McCalla, S.G. Crime modeling with Lévy flights. SIAM Journal On Applied Mathematics, 73(4), 1703-1720, (2013). [CrossRef]

[7] Kolokolnikov, T., Ward, M. \& Wei, J. The stability of steady-state hot-spot patterns for a reaction-diffusion model of urban crime. Discrete And Continuous Dynamical Systems - Series B, 19, 1373, (2014). [CrossRef]

[8] Zipkin, J.R., Short, M.B. \& Bertozzi, A.L. Cops on the dots in a mathematical model of urban crime and police response. Discrete And Continuous Dynamical Systems - Series B, 19(5), 1479, (2014). [CrossRef]

[9] Camacho, A., Lee, H.R.L \& Smith, L.M. Modelling policing strategies for departments with limited resources. European Journal Of Applied Mathematics, 27(3), 479-501, (2016). [CrossRef]

[10] Gu, Y., Wang, Q., \& Yi, G. Stationary patterns and their selection mechanism of urban crime models with heterogeneous near-repeat victimization effect. European Journal of Applied Mathematics, 28(1), 141-178, (2017). [CrossRef]

[11] Pan, C., Li, B., Wang, C., Zhang, Y., Geldner, N., Wang, L. \& Bertozzi, A.L. Crime modeling with truncated Lévy flights for residential burglary models. Mathematical Models And Methods In Applied Sciences, 28(09), 1857-1880, (2018). [CrossRef]

[12] Wang, Q., Wang, D. \& Feng, Y. Global well-posedness and uniform boundedness of urban crime models: One-dimensional case. Journal Of Differential Equations, 269(7), 6216-6235, (2020). [CrossRef]

[13] Rodríguez, N. \& Winkler, M. Relaxation by nonlinear diffusion enhancement in a two-dimensional cross-diffusion model for urban crime propagation. Mathematical Models And Methods In Applied Sciences, 30(11), 2105-2137, (2020). [CrossRef]

[14] Kang, K., Kolokolnikov, T. \& Ward, M. The stability and dynamics of a spike in the 1D Keller-Segel model. IMA Journal Of Applied Mathematics, 72(2), 140-162, (2007). [CrossRef]

[15] Mei, L. \& Wei, J. The existence and stability of spike solutions for a chemotax is system modeling crime pattern formation. Mathematical Models And Methods In Applied Sciences, 30(09), 1727-1764, (2020). [CrossRef]

[16] Kondo, S. \& Miura, T. Reaction-Diffusion Model as a Framework for Understanding Biological Pattern Formation. Science, 329(5999), 1616-1620, (2010). [CrossRef]

[17] Keller, E. \& Segel, L. Traveling bands of chemotactic bacteria: A theoretical analysis. Journal of Theoretical Biology, 30(2), 235-248, (1971). [CrossRef]

[18] Biler, P. \& Wu, G. Two-dimensional chemotaxis models with fractional diffusion. Mathematical Methods In The Applied Sciences, 32(1), 112-126, (2009). [CrossRef]

[19] Calvó-Armengol, A. \& Zenou, Y. Social Networks and Crime Decisions: The Role of Social Structure in Facilitating Delinquent Behavior. International Economic Review, 45(3), 939-958, (2004). [CrossRef] 
[20] Nec, Y. Spike-Type Solutions to One Dimensional Gierer-Meinhardt Model with Lévy Flights. Studies In Applied Mathematics, 129(3), 272-299, (2012). [CrossRef]

[21] Cruz-García, S., Martínez-Farías, F., Santillán-Hernández, A. \& Rangel, E. Mathematical home burglary model with stochastic long crime trips and patrolling: Applied to Mexico City. Applied Mathematics And Computation, 396, 125865, (2021). [CrossRef]

[22] Levajkovic, T., Mena, H. \& Zarfl, M. Lévy processes, subordinators and crime modeling. Novi Sad Journal Of Mathematics, 46(2), 65-86, (2016).

[23] Cohen, L. \& Felson, M. Social Change and Crime Rate Trends: A Routine Activity Approach. American Sociological Review, 44, 588-608, (1979). [CrossRef]

[24] Wilson, J.Q. \& Kelling, G. L. Broken windows. The Atlantic, 249, 29-38, (1982).

[25] Kilbas, A.A., Srivastava, H.M. \& Trujillo, J. Theory and Applications of Fractional Differential Equations (Vol. 204). Elsevier, North-Holland Mathematics Studies, (2006).

[26] Kopriva, D. Implementing Spectral Methods for Partial Differential Equations. Springer, (2009).

[27] Osgood, B.G. Lectures on the Fourier Transform and Its Applications. SIAM, (2019).

[28] Trefethen, L.N. Spectral methods in MATLAB, volume 10 of Software, Environments, and Tools. Society for Industrial and Applied Mathematics (SIAM), Philadelphia, PA, 24, (2000).

[29] Butcher, J. Numerical Methods for Ordinary Differential Equations. John Wiley, 2015.

[30] Ghrist, M.L., Fornberg, B. \& Reeger, J.A. Stability ordinates of Adams predictor-corrector methods. BIT Numerical Mathematics, 55(3), 733-750, (2015). [CrossRef]

[31] Lindfield, G.R. \& Penny, J.E. Numerical methods: using MATLAB. Elsevier, 2012.

[32] Tadjeran, C. \& Meerschaert, M.M. A second-order accurate numerical method for the two-dimensional fractional diffusion equation. Journal of Computational Physics, 220(2), 813-823, (2007). [CrossRef]

[33] Lapidus, L. \& Seinfeld, J. 4 Predictor-Corrector Methods. Numerical Solution Of Ordinary Differential Equations, 74, 152-241, (1971).

[34] Rodriguez, N., \& Bertozzi, A. Local existence and uniqueness of solutions to a PDE model for criminal behavior. Mathematical Models and Methods in Applied Sciences, 20(supp01), 1425-1457, (2010). [CrossRef]

[35] Cantrell, R.S., Cosner, C. \& Manásevich, R. Global Bifurcation of Solutions for Crime Modeling Equations. SIAM Journal On Mathematical Analysis, 44(3), 1340-1358, (2012). [CrossRef]

[36] Tse, W.H. \& Ward, M.J. Hotspot formation and dynamics for a continuum model of urban crime. European Journal OfApplied Mathematics, 27(3), 583-624, (2016). [CrossRef]

[37] Rodríguez, N., Wang, Q. \& Zhang, L. Understanding the Effects of On- and Off-Hotspot Policing: Evidence of Hotspot, Oscillating, and Chaotic Activities. SIAM Journal On Applied Dynamical Systems, 20(4), 1882-1916, (2021). [CrossRef]

Mathematical Modelling and Numerical Simulation with Applications (MMNSA) (https://www.mmnsa.org)

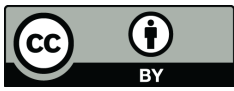

Copyright: ( 2022 by the authors. This work is licensed under a Creative Commons Attribution 4.0 (CC BY) International License. The authors retain ownership of the copyright for their article, but they allow anyone to download, reuse, reprint, modify, distribute, and/or copy articles in MMNSA, so long as the original authors and source are credited. To see the complete license contents, please visit (http://creativecommons.org/licenses/by/4.0/). 